\title{
ABUNDANCES IN THE GASEOUS GALACTIC HALO
}

\author{
ULYSSES J. SOFIA \\ Villanova University \\ Department of Astronomy and Astrophysics, Villanova, PA 19085 USA
}

\begin{abstract}
The well measured gas-phase abundances in the low halo suggest that this region of the Galaxy has total (gas plus dust) metal abundances which are close to those in the solar neighborhood. The gas-phase abundances in the halo are generally higher than those seen in the disk, however, this affect is likely due to the destruction of dust in the halo clouds. Observations of high velocity clouds (HVCs) in the halo suggest that these clouds have metal abundances which are substantially lower than those measured for the local interstellar medium. These determinations, however, are often of lower quality than those for the low halo because of uncertainties in the hydrogen abundances along the sightlines, in the incorporation of elements into dust, and in the partial ionization of the clouds.
\end{abstract}

\section{Introduction}

The abundance of elements in the gas and dust phases of the Galactic disk have been well studied. Data obtained with the Copernicus satellite in the 1970's gave us much insight into interstellar medium (ISM) (Jenkins 1987). The Goddard High Resolution Spectrograph (GHRS) aboard the Hubble Space Telescope (HST) has refined these studies through its enhanced resolution over Copernicus. This better allows individual absorbing clouds to be studied rather than average line of sight properties (Spitzer \& Fitzpatrick 1993, Sofia, Cardelli \& Savage 1994).

Halo gas studies naturally lagged behind exploration of the Galactic disk. The distance to the sources are greater and the velocity structure of the absorption is needed to distinguish the halo from the disk gas. Studies of halo abundances are important for exploring a region of the Galaxy which appears to differ greatly from the disk. The stellar population of the halo is quite different from the disk suggesting that the beginning and end products of stellar lifecycles (the ISM) are different (Jenkins \& Wallerstein 1996). Also, differences in the kinematics (Danly et al. 1992) and ionization characteristics (Savage \& Massa 1987) lead one to conclude that the ISM in the halo may differ from that in the disk.

Studies of elemental abundances in halo gas have greatly progressed since the launch of HST in 1990. The GHRS aboard the HST allowed ultraviolet absorption features toward halo stars to be observed at a resolution of $3.5 \mathrm{~km} \mathrm{~s}^{-1}$. Since halo gas along a sightline can be separated from the local gas through differences in radial velocities, the. high-resolution GHRS spectra allow gas from the halo to be well isolated. High resolution spectra also have the advantage of determining if, and possibly how much, saturation is affecting an absorption feature (Savage \& Sembach 1991, Jenkins 1996). The advantage of HST's ultraviolet spectral coverage is that this region contains a large number of resonance transitions of species dominant in the neutral ISM.

\section{The Low Halo}

Sembach \& Savage (1996) have studied the abundance of 8 elements in the low halo ISM in 10 absorbing regions toward 6 Galactic and 1 extragalactic sources. The data which they use are original as well as from Sofia, Savage \& Cardelli (1993), Spitzer \& Fitzpatrick (1993, 1995), Savage et al. (1992, 1993), Savage \& Sembach (1995), Cardelli et al. $(1991,1994,1995)$ and Sofia et al. (1994). The elements studied varied in their dust incorporation characteristics from extremely low 
depletions (sulfur) to moderate ( $\mathrm{Mg}, \mathrm{Si}$ and $\mathrm{Mn}$ ) and highly depleted ( $\mathrm{Cr}, \mathrm{Fe}, \mathrm{Ni}$ and $\mathrm{Ti}$ ) species. They find that the low-halo gas abundances are generally higher than the disk abundances when compared to zinc. Zinc is a low depletion species (Roth \& Blades 1995, Sembach et al. 1995) and can be used as a proxy for hydrogen. The apparently greater metal abundances in the halo could either reflect a higher overall abundance of the elements, or less depletion onto interstellar grains in the halo.

Figure 1 shows the logarithmic abundances in the 7 components of 5 sightlines which Sembach \& Savage believe contain only halo gas (not all elements are available for each absorption component). The quantity plotted is the logarithmic abundance ratio in the cloud compared to the cosmic abundance ratio or

$$
\left[\frac{X}{H}\right] \sim\left[\frac{X}{Z n}\right]=\log \left[\frac{N(X)}{N(Z n I I)}\right]-\log \left(\frac{X}{Z n}\right)_{\text {cosmic }} \text {. }
$$

The measurement errors are also plotted for each data point. The adopted cosmic abundances in the Figure are from solar ratios, although it is not clear that this is appropriate (Sofia et al 1994, Snow \& Witt 1996, Fitzpatrick 1996). It is obvious from the Figure that the abundances of the elements relative to $\mathrm{Zn}$ (or hydrogen) are quite uniform for the low halo. The solid squares represent the weighted average for each element in the halo sightlines. It is unlikely that the abundance ratios would be so uniform if the intrinsic interstellar abundances (i.e. gas + dust) were not the same for all of the sightlines.

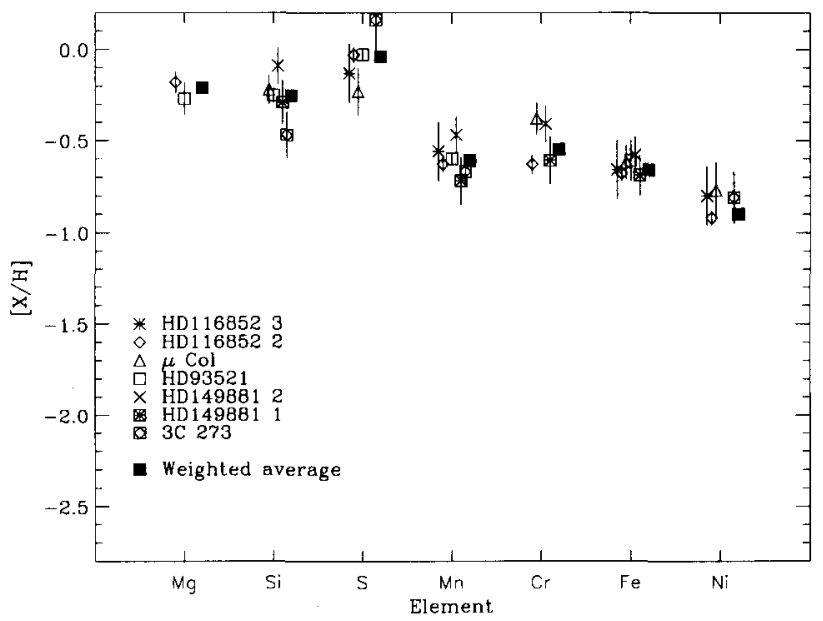

Figure 1. Logarithmic abundance ratios for low halo sightlines as compared to the solar abundance ratios.

The mean values plotted for the relative logarithmic halo abundances are listed in Table 1 for cosmic abundances represented by the sun and by the ISM values suggested by Snow \& Witt (1996). It is clear from the Table that either of the cosmic abundance scenarios produces depletions which can be reasonably explained by dust incorporation of the missing atoms. Sembach \& Savage (1996) in fact argue that the depletions in these clouds likely represent the composition of resilient dust grain cores. This is supported by the smooth transition of depletion patterns from disk to halo sightlines (see Figure 6 in Sembach \& Savage 1996).

What is likely occurring in the low halo is that the composition of the ISM is very much like that in the Galactic disk. This may not be surprising since neutral $\mathrm{H}$ observations show that the turbulent motions of the ISM may well mix the disk and low halo (Lockman \& Gehman 1991). 


\begin{tabular}{lcc}
$\begin{array}{l}\text { TABLE 1. } \\
\text { Abundances }\end{array}$ & Measured & Relative Halo \\
\hline Element & {$[\mathrm{X} / \mathrm{H}]_{\text {eun }}$} & {$[\mathrm{X} / \mathrm{H}]_{\text {ISM }}$} \\
\hline $\mathrm{Mg}$ & -0.21 & -0.02 \\
$\mathrm{Si}$ & -0.25 & +0.03 \\
$\mathrm{~S}$ & -0.04 & +0.14 \\
$\mathrm{Mn}$ & -0.61 & $?$ \\
$\mathrm{Cr}$ & -0.55 & -0.38 \\
$\mathrm{Fe}$ & -0.66 & -0.38 \\
$\mathrm{Ni}$ & -0.90 & -0.70 \\
\hline
\end{tabular}

\section{High Velocity Clouds}

High velocity clouds (HVCs) in the halo may represent a population of ISM gas which has been isolated from the low halo and disk material, and therefore may not contain the same elemental abundances. There are problems with HVC abundance measurements, however. As with the low halo, in order to derive accurate ISM abundances one must be aware of phenomena which can mimic apparent changes in metallicity.

Observations of HVCs with abundances which are apparently as low as $10 \%$ of the solar values are extremely intriguing and suggestive of unenriched material. The magnitude of the pitfalls in the metallicity determinations, however, is often difficult to quantify. For instance Bowen \& Blades (1993) suggest that the low metal abundance they see in the HVC toward Mrk 205 may be partially or completely an ionization effect. Schwartz, Wakker \& van Woerden (1995) discuss the possibility that depletion of material onto dust grains may significantly affect the interpretation of abundances. Lu, Savage \& Sembach (1994) have abundance measurements in an HVC toward NGC 3783. They minimize depletion effects by observing sulfur (Spitzer \& Fitzpatrick 1993) and of ionization effects by comparing S II to H I (Savage et al 1993). They find a sulfur-to-hydrogen ratio that is approximately $0.15 \pm 0.05$ that in the Sun, but question the reliability of the neutral $H$ abundance used for the comparison. Their $\mathrm{H} \mathrm{I}$ abundance was derived from $21 \mathrm{~cm}$ emission spectra. Radio beams are large relative to the stellar point source used for absorption studies, therefore they will measure $\mathrm{H} \mathrm{I}$ outside of the line of sight. Since large variations of $\mathrm{H} \mathrm{I}$ column densities occur over small (arcminute) regions within an HVC (Wakker \& Schwartz 1991), using $21 \mathrm{~cm}$ column densities may lead to inaccurate conclusions.

\section{Conclusions}

The abundances that we can measure well, those in the low halo, suggest that composition in this region is much like that of the local ISM. HVCs, which we know are unusual by virtue of their radial velocities, often appear to have metallicities which are a small fraction of the local ISM or the Sun. These clouds may indeed be extremely subsolar in abundances, however they are intrinsically difficult to measure. One must be aware of of the limitations of these studies and interpret the results accordingly.

\section{References}

Bowen, D.V. and Blades, J.C. 1993, $A p J, 403$, L55

Cardelli, J.A., Savage, B.D., Bruhweiler, F.C., Smith, A.M., Ebbets, D.C., Sembach, K.R. and Sofia, U.J. 1991, ApJ, 377, L57

Cardelli, J.A., Savage, B.D., Sofia, U.J., Keenan, F.P. and Dufton, P.L. 1994, ApJ, 420, L29

Cardelli, J.A., Sembach, K.R. and Savage, B.D. 1995, ApJ, 440, 221

Danly, L., Lockman, F.J., Meade, M. and Savage, B. D. 1992, ApJS, 81, 125

Fitzpatrick, E.L. 1996, A pJ, 473, L55

Jenkins, E.B. 1987, in Interstellar Processes, eds. D.J. Hollenbach and H. A. Thronson, Jr., Klewer Academic Publishers, Dordrecht, 533 
Jenkins, E.B. $1996, A p J, 471,292$

Jenkins, E.B. and Wallerstein, G. 1996, ApJ, 462, 758

Lockman, F.J. and Gehman, C.S. 1991, ApJ, 382, 182

Lu, L., Savage, B.D. and Sembach, K.R. 1994, ApJ, 426, 563

Roth, K.C. and Blades, J.C. 1995, ApJ, 445, L95

Savage, B.D., Cardelli, J.A. and Sofia, U.J. 1992, $A p J, 401,706$

Savage, B.D., Lu, L., Weymann, R.J., Morris, S.L. and Gilliland, R. 1993, ApJ 404, 124

Savage, B.D. and Massa, D. 1987, ApJ, 314, 380

Savage, B.D. and Sembach, K.R. 1991, ApJ, 379, 245

$-1995, A p J, 434,145$

Schwartz, U.J., Wakker, B.P. and van Woerden, H. 1995, $A \& A, 302,364$

Sembach, K.R. and Savage, B.D. 1996, ApJ. 457, 211

Sembach, K.R., Steidel, C.C., Macke, R.J. and Meyer, D.M. 1995, ApJ, 445, L27

Snow, T.P. and Witt, A.N. $1996, A p J, 468$, L65

Sofia, U.J., Cardelli, J.A. and Savage, B.D. 1994, ApJ, 430, 650

Sofia, U.J., Savage, B.D. and Cardelli, J.A. 1993, $A p J, 413,251$

Spitzer, L. and Fitzpatrick, E.L. 1993, ApJ, 409, 299

$-, 1995, A p J, 445,196$

Wakker, B.P. and Schwartz, U.J. 1991, $A \& A, 250,484$ 